\title{
The prevalence of celiac disease, related HLA-genotypes, and comorbidities among Egyptian girls with Turner syndrome: a single-centre study
}

\author{
Nanees Abdel-Badie Salem', Shimaa R. Hendawy² \\ 'Pediatric Endocrinology and Diabetes Unit, Department of Pediatrics, Faculty of Medicine, Mansoura University, Egypt \\ ${ }^{2}$ Department of Clinical Pathology, Mansoura University, Egypt
}

\section{ABSTRACT}

\begin{abstract}
Aim of the study: An association between celiac disease (CD) and Turner syndrome (TS) has gained widespread recognition with wide-ranging prevalence rates. Insufficient data are available concerning this association among Egyptian TS girls. Therefore, we aimed to examine the prevalence of $\mathrm{CD}$; to define the predisposing HLA-genotypes, clinical characteristics and associated comorbidities in a cohort of Egyptian TS girls; and to assess the impact of karyotypes and oestrogen exposure on the overall prevalence of autoimmunity.

Material and methods: Fifty-five TS girls were initially screened by total IgA and anti-tissue transglutaminase (anti-tTG) IgA. Anti-tTG-IgG assay is a second step in IgA-deficient girls. CD-seropositive TS girls were subjected to HLA-typing and endoscopic duodenal biopsies, and were evaluated for associated comorbidities. Results: Seroprevalence (anti-tTG-IgA > $10 \mathrm{U} / \mathrm{ml}$ ) and biopsy-confirmed prevalence of CD were 5.5\% (3/55) and 3.6\% (2 girls only underwent endoscopic biopsies and displayed Marsh-IIIb), respectively. Absolute and partial IgA-deficiency were observed in 1 and 5 girls, respectively; they were all negative for anti-tTG-IgG. HLA-typing of CD-seropositive girls showed that case 1: DQA1+/DRB1-; case 2: DQA1+/DRB1+; and case 3: DQB1+/DRB1-. Iron-deficiency anaemia, vitamin-D deficiency, and low bone mineral density were detected among celiac girls. No significant associations were observed between different karyotypes or oestrogen exposure and autoimmunity prevalence.

Conclusions: Seroprevalence and biopsy-confirmed prevalence of CD in Egyptian TS girls were 5.5\% and $3.6 \%$, respectively, which supports the association between these disorders and reinforces the importance of screening for CD in TS patients as a high-risk population. All CD-seropositive girls displayed the predisposing CD HLA-DQ2 and/or DR4 alleles. Careful surveillance for comorbidities is essential to improve overall health outcomes.
\end{abstract}

KEY WORDS:

celiac disease, immunoglobulin A, Turner syndrome, autoimmune disorders.

\section{INTRODUCTION}

Turner syndrome (TS) results from numeric or structural abnormalities of the second X-chromosome, affecting 25-50/100,000 females [1]. Individuals with TS are at high risk for developing autoimmune disorders (AD) with preponderance towards autoimmune thyroiditis (AIT) and type 1 diabetes mellitus (T1DM), and less frequently inflammatory bowel and rheumatic diseases [2-6]. The prevalence of $\mathrm{AD}$ evolves with age $[5,6]$, and more than one $\mathrm{AD}$ can coexist $[3,4]$.

Celiac disease (CD) is an immune-mediated enteropathy triggered by gluten ingestion in genetically susceptible individuals. CD has strongly associated with human leu-

\section{ADDRESS FOR CORRESPONDENCE:}

Nanees Abdel-Badie Salem, Pediatric Endocrinology and Diabetes Unit, Department of Pediatrics, Faculty of Medicine, Mansoura University, Egypt, e-mail: nanees.salem@gmail.com 
cocyte antigen (HLA) class-II; almost all celiac patients ( $95 \%)$ carry genes coding for DQ2 $\alpha / \beta$ heterodimer, and the remaining $D Q 2$-negative celiac patients display DR4-DQ8 haplotype, which facilitates CD4 ${ }^{+} \mathrm{T}$-cell recognition of gluten-derived peptides $[7,8]$. CD has wide clinical presentations, classic gastrointestinal or non-gastrointestinal symptoms, or is asymptomatic in high-risk individuals. Duodenal biopsy is the gold standard for CD diagnosis; however, serological tests including anti-tissue transglutaminase (anti-tTG) IgA assay are recommended in the initial screening for $\operatorname{CD}[7,8]$.

More than 3 decades have elapsed since the first description of CD in a TS patient [9]; thereafter, the association between $\mathrm{CD}$ and TS has gained widespread recognition with wide-ranging prevalence rates [10-15]. The underlying mechanisms for increased risk of autoimmunity in TS remain unclear. Haploinsufficiency of immunoregulatory genes located on X-chromosome, e.g. X-linked forkhead box-P3 (FOXP3) gene [16], and oestrogen therapy [17] were hypothesized as contributing factors. However, the role of FOXP3 in CD pathogenesis is not documented [18].

Insufficient data are available concerning the association between CD and TS in Egypt. We aimed to examine the prevalence of $C D$ with surveillance for $C D$ predisposing HLA-genotyping and CD-related comorbidities and autoimmunity in a cohort of Egyptian girls with TS, and to assess the impact of karyotypes and oestrogen exposure on the overall prevalence of autoimmunity in TS girls.

\section{MATERIAL AND METHODS}

This was a cross-sectional study conducted between March 2019 and March 2020. Fifty-five TS girls diagnosed by karyotyping of peripheral lymphocytes were recruited sequentially during their routine visits at the Paediatric Endocrinology Clinic at Mansoura University Children's Hospital.

The study was approved by the Ethics Committee of Mansoura University, Faculty of Medicine - Institutional Research Board (IRB) (Code No. R.20.04.800). Informed consent was obtained from parents of all participants included in the study

A detailed questionnaire was compiled with particular attention paid to CD-related symptoms such as chronic diarrhoea/diarrheic bouts, vomiting, abdominal pain/ distension, constant tiredness, and weight loss. History of previously diagnosed AD was obtained. History of growth hormone therapy (GHT) and exposure to oestrogen, either spontaneous breast development or oestrogen replacement therapy (ERT), were addressed. Height and weight Z-scores were calculated according to Egyptian TS growth charts [19].

At time of screening for $\mathrm{CD}$, all girls were on a gluten-containing diet, and none had been previously diag- nosed with CD. Five-millilitre venous samples were collected from all participants, centrifuged, and sera were stored at $-20^{\circ} \mathrm{C}$ until time of analysis.

Initial screening was conducted by combined total IgA and anti-tTG-IgA assays. In the case of IgA deficiency, an anti-tTG-IgG assay was performed.

Total serum IgA was measured using a Human IgA enzyme linked immunosorbent assay (ELISA) kit (Bioassay Technology Laboratory, Cat. No. E0189Hu, Shanghi, China). Absolute IgA deficiency is defined as serum IgA $<5 \mathrm{mg} / \mathrm{dl}$, while partial IgA deficiency refers to serum IgA $>5 \mathrm{mg} / \mathrm{dl}$ but more than 2 standard deviations (SD) below age-adjusted means [20]. Age-adjusted values for serum IgA were specified in the $18^{\text {th }}$ edition of Nelson Textbook of Paediatrics as follows; $2-5$ years: $14-159 \mathrm{mg} / \mathrm{dl}$; 6-10 years: $33-236$ $\mathrm{mg} / \mathrm{dl}$; and 11-18 years: $70-312 \mathrm{mg} / \mathrm{dl}$ [21].

Serum anti-tTG-IgA was measured using ELISA kit (Orgentec Diagnostika GmbH, Mainz, Germany) with a lower detection limit of $1.0 \mathrm{U} / \mathrm{ml}$ and a cut-off point for a positive result of $10 \mathrm{U} / \mathrm{ml}$. Seropositive patients with anti-tTG-IgA $\geq 3$ times the upper normal limit ( $10 \mathrm{U} / \mathrm{ml})$ were offered endoscopic duodenal biopsies to confirm CD diagnosis according to the European Society of Paediatric Gastroenterology, Hepatology, and Nutrition criteria [8]. Mucosal sections were evaluated according to Marsh-Oberhuber grading, where Marsh-I; > 25 intraepithelial-lymphocytes/100 enterocytes, Marsh-II; increased intraepithelial-lymphocytes with crypt hyperplasia, and partial, subtotal or total villi atrophy classified as Marsh IIIa, IIIb, and IIIc, respectively [22].

Genomic DNA were extracted from peripheral blood lymphocytes of girls showing positivity on serological testing and purified according to the manufacturer's instructions using a commercial kit (GFX Genomic Blood Purification KIT, Amersham Biosciences, USA) and then subjected to polymerase chain reaction technique (PCR) with specific primers for the presence of CD predisposing HLA alleles coded by $D Q A 1^{\star} 0501, D Q B 1^{\star} 0201$, and $D R B 1^{*} 04$ by applying the method described by Sacchetti et al. [23].

Seropositive TS girls were further evaluated for CD-comorbidities including the following: haemoglobin, transferrin saturation, alanine/aspartate aminotransferases, serum calcium, phosphorus, alkaline phosphatase, parathormone, and 25-hydroxy vitamin D levels. Bone mineral density (BMD) for total body and lumbar spine (L1-L4) were evaluated by dual-energy X-ray absorptiometry (DXA) scan, and the BMD Z-score was interpreted as either at risk for low BMD "BMD Z-score: -1.0 and -1.9 " or low BMD with increased fracture risk "BMD Z-score $\leq-2.0$ " [24].

\section{STATISTICAL ANALYSIS}

Data were analysed using SPSS software version 16.0 (SPSS Inc., Chicago, USA). Qualitative data were 
presented as frequencies [number (\%)]. Quantitative data were presented as median (minimum-maximum) for non-parametric data and as mean \pm SD for parametric data after testing normality using the Kolmogorov-Smirnov test. Age groups were compared using Student's t-test. Distributions among groups were tested by Fisher's exact test. Pearson correlation was used to study the relation between age at oestrogen exposure and age at autoimmunity diagnosis. $P<0.05$ was considered significant.

\section{RESULTS}

The mean age at TS diagnosis was $6.2 \pm 2.3$ years and at screening for CD it was $12.6 \pm 3.2$ years (range: $4.5-18$ ), with significant lag in initiation of the screening for CD among the current TS cohort $(p<0.001)$. Demographic data of the study population are presented in Table 1.

Karyotypes distribution among TS girls were as followis: 45,X $(n=27)$; structural aberrations $(n=18)$ and mosaicism $(n=10)$ (Table 2$)$.

Forty-seven girls are currently receiving GHT, $0.15 \mathrm{IU} / \mathrm{kg} /$ day subcutaneously, and 8 girls had stopped GHT at a mean age of $14.8 \pm 0.6$ years when height velocity was $<3 \mathrm{~cm} /$ year. ERT was initiated for 23 girls who had exhibited no clinical signs of spontaneous puberty by 14 years of age, and 4 girls had spontaneous puberty at a mean age of $13.9 \pm 0.7$ years.

\section{SERUM IGA STATUS IN TS GIRLS}

Serum IgA was within the normal range in $89.1 \%$ (49/55) of girls. Absolute IgA-deficiency $(<5 \mathrm{mg} / \mathrm{dl})$ was observed in 1 girl (1.8\%), while partial IgA-deficiency was observed in 5 girls (9.1\%). All IgA-deficient girls were anti-tTG-IgG negative $(<10 \mathrm{U} / \mathrm{ml})$. A TS girl who had IgA-deficiency reported recurrent otitis and sinusitis. None of the IgA-deficient girls had other ADs. Table 3 showed the characteristics of IgA-deficient girls.

\section{PREVALENCE OF CD IN TS GIRLS}

Three out of 55 TS girls showed seropositivity to anti-tTG-IgA, of whom 2 girls only had anti-tTG-IgA titre $\geq 3$ times the cut-off value (case 1: $189 \mathrm{U} / \mathrm{ml}$ and case 2: $73.4 \mathrm{U} / \mathrm{ml}$ ) and underwent duodenal endoscopic biopsies after their parents' approval. The histopathology was compatible with Marsh grade-IIIb. In case 3, anti-tTGIgA was slightly elevated $(15.7 \mathrm{U} / \mathrm{ml})$.

Therefore, the sero-prevalence (anti-tTG-IgA > $10 \mathrm{U} / \mathrm{ml}$ ) and biopsy-confirmed prevalence of CD was 5.5\% (3/55)

TABLE 1. General characteristics and serology results of the Turner syndrome girls $(n=55)$.

\begin{tabular}{|c|c|}
\hline Parameters & Value \\
\hline Age at TS diagnosis (years) (mean \pm SD) & $6.2 \pm 2.3$ \\
\hline Age at screening for $C D$ (years) (mean $\pm S D$ ) & $12.6 \pm 3.2$ \\
\hline \multicolumn{2}{|l|}{ Current medications [number (\%)] } \\
\hline GH therapy & $47(85.5)$ \\
\hline Oestrogen replacement therapy & $23(41.8)$ \\
\hline \multicolumn{2}{|l|}{ Serology for Celiac disease [number (\%)] } \\
\hline Seropositive (anti-tTG $\lg A \geq 10 \mathrm{U} / \mathrm{ml}$ ) & $3(5.5)$ \\
\hline Seronegative (anti-tTG $\lg \mathrm{A}<10 \mathrm{U} / \mathrm{ml}$ ) & $52(94.5)$ \\
\hline \multicolumn{2}{|l|}{ Total IgA level [number (\%)] } \\
\hline Normal IgA level & $49(89.1)$ \\
\hline $\operatorname{lgA}$ deficiency $(<5 \mathrm{mg} / \mathrm{dl})$ & $1(1.8)$ \\
\hline Partial IgA deficiency & $5(9.1)$ \\
\hline \multicolumn{2}{|l|}{ Frequency of autoimmune diseases [number (\%)] } \\
\hline Biopsy-proven CD & $2(3.6)$ \\
\hline AIT (isolated) & $4(7.2)$ \\
\hline CD-Seropositivity + AIT & $1(1.8)$ \\
\hline Type 1 diabetes mellitus & $1(1.8)$ \\
\hline Scalp psoriasis & $1(1.8)$ \\
\hline Overall prevalence of autoimmune diseases & $9(16.4)$ \\
\hline
\end{tabular}

AlT - autoimmune thyroiditis; anti-tTG - anti-tissue transglutaminase; IgA - immunoglobulin A; $C D$ - celiac disease

TABLE 2. Distribution of karyotype in Turner syndrome girls $(n=55)$ and in relation to the autoimmune disorders

\begin{tabular}{|c|c|c|c|c|c|c|c|}
\hline & $\begin{array}{l}\text { Turner patients } \\
\quad(n=55)\end{array}$ & $\begin{array}{c}\text { Overall } \\
\text { autoimmunity }\end{array}$ & CD & AIT & $\mathrm{CD}+\mathrm{AlT}$ & T1DM & Psoriasis \\
\hline Monosomy 45,X ( $n=27)$ & $27(49 \%)$ & $6 / 27(22.2 \%)$ & $1 / 27$ & $3 / 27$ & $1 / 27$ & $1 / 27$ & - \\
\hline \multicolumn{8}{|l|}{ Structural abnormality $(n=18)$} \\
\hline Isochromosome 46,X,i[Xq10] & $14(25.5 \%)$ & $1 / 14(7.1 \%)$ & $1 / 14$ & - & - & - & - \\
\hline Deletion 46,X,del(Xp-) & $4(7.3 \%)$ & & - & - & - & - & - \\
\hline \multicolumn{8}{|l|}{ Mosaicism $(n=10)$} \\
\hline $45, X / 46, X X$ & $6(11 \%)$ & $1 / 6(16.7 \%)$ & - & $1 / 6$ & & - & - \\
\hline $45, X / 46, X, i[X q 10]$ & $3(5.5 \%)$ & $1 / 3(33.3 \%)$ & - & - & & - & $1 / 3$ \\
\hline $45, X / 46, X,+$ marker & $1(1.8 \%)$ & - & - & - & & - & - \\
\hline
\end{tabular}

AlT - autoimmune thyroiditis; $C D$ - celiac disease; T1DM - type 1 diabetes mellitus 
TABLE 3. Characteristics of Turner syndrome girls with IgA deficiency $(n=6)$

\begin{tabular}{|l|c|c|c|c|c|}
\hline & $\begin{array}{c}\text { Age } \\
\text { (years) }\end{array}$ & Cytogenetic & $\begin{array}{c}\text { Serum IgA } \\
(\mathrm{mg} / \mathrm{dl})\end{array}$ & $\begin{array}{c}\text { Anti-tTG IgA } \\
(\mathrm{U} / \mathrm{ml})\end{array}$ & $\begin{array}{c}\text { Anti-tTG IgG } \\
(\mathrm{U} / \mathrm{ml})\end{array}$ \\
\hline Absolute IgA deficiency $(<5 \mathrm{mg} / \mathrm{dl})(n=1)$ & 9.9 & $46, \mathrm{X},[\mathrm{Xq} 10]$ & 4.3 & 0.18 & 2.6 \\
\hline \begin{tabular}{l} 
Partial IgA deficiency $(n=5)$ \\
\hline
\end{tabular} & 9.5 & $45, \mathrm{X}$ & 8.2 & 0.3 & 1.7 \\
\hline & 10 & $45, \mathrm{X}$ & 12.3 & 0.78 & 2.2 \\
\hline & 12 & $45, \mathrm{X} / 46, \mathrm{XX}$ & 10.4 & 2.4 & 1.9 \\
\hline & 14.5 & $45, \mathrm{X}$ & 18.8 & 1.3 & 0.5 \\
\hline & 15 & $45, \mathrm{X}$ & 16.4 & 0.8 & 3.1 \\
\hline
\end{tabular}

anti-tTG - anti-tissue transglutaminase; IgA - immunoglobulin A; lgG - immunoglobulin $G$

TABLE 4. Characteristics of Turner syndrome girls with celiac disease seropositivity

\begin{tabular}{|c|c|c|c|}
\hline Parameter & Case 1 & Case 2 & Case 3 \\
\hline Karyotype & $45, X$ & $46, X, i[X q 10]$ & $45, X$ \\
\hline Age at TS diagnosis (years) & 6.5 & 8 & 11.3 \\
\hline Age at CD diagnosis (years) & 8.5 & 11 & 14 \\
\hline CD-related symptoms & Abdominal pain/ distension & Diarrheic bouts weight loss & Asymptomatic \\
\hline Anti-tTG lg A titre (U/ml) & 189 & 73.4 & 15.7 \\
\hline Total $\lg A(\mathrm{mg} / \mathrm{dl})$ & 96.5 & 127 & 178.8 \\
\hline HLA-genotype & DQA1+/DRB1- & DQA1+/DRB1+ & DQB1+/DRB1- \\
\hline Histopathology of duodenal biopsy & Marsh IIlb & Marsh IIllb & Not done \\
\hline Height $(\mathrm{cm})$ & 103 & 117 & 133 \\
\hline Height z-score & $-2.2\left(<3^{\text {rd }}\right.$ percentile $)$ & -1.31 & -0.22 \\
\hline Weight (kg) & 19.5 & 20.5 & 40 \\
\hline Weight z-score & -2.3 & -3.0 & -2.2 \\
\hline Haemoglobin ( $\mathrm{g} / \mathrm{dl})$ & 11.3 & 10.2 & 12.8 \\
\hline Transferrin saturation (15-50\%) & $13.3 \%$ & $10.5 \%$ & $34 \%$ \\
\hline Serum $25-0 \mathrm{H}$ vitamin D $(20-100 \mathrm{ng} / \mathrm{ml})$ & 16.54 & 12.39 & 15.7 \\
\hline Serum parathormone (15-65 pg/ml) & 110.1 & 81.6 & 75 \\
\hline \multicolumn{4}{|l|}{ DXA scan } \\
\hline Lumbar spine (L1-L4) BMD Z-score & -1.4 & -1.7 & -2.6 \\
\hline Total body BMD Z-score & -1.3 & -1.5 & -2.5 \\
\hline
\end{tabular}

anti-tTG-IgA - anti-tissue transglutaminase immunoglobulin A; BMD - bone mineral density; CD - celiac disease; DEXA - dual-energy X-ray absorptiometry; HLA - human leucocyte antigen; IgA - immunoglobulin A; TS Turner syndrome

and 3.6\% (2 girls only fulfilled criteria for endoscopy out of 55), respectively.

\section{RESULTS OF CD-RELATED HLA-GENOTYPING OF TS GIRLS WITH CD-SEROPOSITIVITY (TABLE 4)}

Results of HLA-typing showed that all CD-seropositive girls presented the predisposing CD HLA-alleles as follows: case 1: $D Q A 1+/ D R B 1-$; case 2: $D Q A 1+/ D R B 1+$; and case $3: D Q B 1+/ D R B 1-$.

\section{CLINICAL AND LABORATORY CHARACTERISTICS OF TS GIRLS WITH CD-SEROPOSITIVITY (TABLE 4)}

Typical CD gastrointestinal symptoms were evident in case 1 and case 2, while case 3 was asymptomatic except for post-meal gastric discomfort.

All seropositive girls were underweight, while only case 1 had stature below the $3^{\text {rd }}$ percentile according to Egyptian TS growth charts. 
Laboratory evaluation revealed iron deficiency anaemia in case 1 and case 2 . In all girls, vitamin D was low, accompanied by elevated parathormone level. Normal serum calcium, phosphorus, alkaline phosphatase, and liver enzymes were detected. Low BMD with increased fracture risk was detected in case 3, while case 1 and case 2 were at risk of low BMD.

\section{OVERALL PREVALENCE OF AUTOIMMUNITY IN TS GIRLS (TABLE 2)}

Five girls (9\%) had AIT, Hashimoto's disease, at a mean age of $12 \pm 2.4$ years. They all were asymptomatic (subclinical hypothyroidism) and none had Graves' disease. In case 3, CD-seropositivity coexisted with AIT diagnosed earlier at 12 years of age. One TS girl (1.8\%), aged 7 years, was diagnosed as T1DM at 5 years of age, and one TS girl (1.8\%), aged 15 years, was diagnosed recently at 14.5 years with scalp psoriasis.

\section{THE IMPACT OF AGE OF TS GIRLS ON AUTOIMMUNITY}

No significant difference was detected in the mean age of TS girls between those with CD-seropositivity and those with CD-seronegativity [11.2 \pm 2.8 versus 12.7 $\pm 3.2 ; p=0.411]$; or between those diagnosed with at least one $\mathrm{AD}$ and those without $[13.1 \pm 2.4$ versus $12.6 \pm 3.3$; $p=0.701]$.

\section{THE IMPACT OF OESTROGEN EXPOSURE ON AUTOIMMUNITY}

Out of the 9 girls with $\mathrm{AD}, 5$ girls (55.6\%) were exposed to oestrogen and 4 girls (44.4\%) were not. No significant correlation was detected between the age at oestrogen exposure $(13.4 \pm 0.7)$ and the age at $\mathrm{AD}$ diagnosis $(12.9 \pm 2.4)(\mathrm{r}=0.602 ; p=0.282)$.

\section{IMPACT OF KARYOTYPE ON AUTOIMMUNITY}

Table 2 shows the distribution of AD among karyotypes. No significant association was detected between 45,X karyotype (6/9 with AD; 66.7\%) and overall prevalence of autoimmunity compared to non-45,X karyotype (3/9 with $\mathrm{AD} ; 33.3 \%)(p=0.503)$.

\section{DISCUSSION}

In the current Egyptian TS cohort $(n=55)$, the seroprevalence of CD (anti-tTG-IgA $>10 \mathrm{U} / \mathrm{ml}$ ) was $5.5 \%(3 / 55)$, being 12 -times higher than that reported in general Egyptian paediatric females $(0.46 \%$; 3 out of 656 girls) [25]. The prevalence rate of biopsy-proven CD was $3.6 \%$ because only 2 girls fulfilled the criteria for en- doscopy. A significant lag in initiation of screening for CD was detected among the current TS cohort.

In a previous Egyptian serology study, 10 out of $80 \mathrm{TS}$ girls were seropositive for anti-tTG IgA, giving a prevalence of $12.5 \%$ [26]. Thus, based on this study (10/80) and the current study (3/55), the total prevalence of CD-seropositivity among Egyptian TS girls could reach 9.6\% $(13 / 135)$, quite a high prevalence indicating that the association between these 2 disorders could not be coincidental.

The prevalence of CD in the current Egyptian TS cohort is comparable to that reported by most of previous screening studies conducted at specialist single centres on small cohorts of paediatric TS populations, where the prevalence of CD among Iranian TS girls was $4.1 \%$ $(2 / 48)$ versus $0.6 \%$ in the general population (GP) [11]; $3.6 \%(2 / 56)$ among Brazilian TS girls versus $0.34 \%$ in GP [12]; and 5.5\% (4/73) among TS girls from Poland versus $0.8 \%$ in paediatric GP [13]. A higher CD prevalence was reported for Irish TS girls (9\%) [14] and for Italian TS girls $(12.9 \%$; 4/31) [15]. The prevalence of CD in TS patients in multicentre studies conducted in Sweden, Canada, Poland, and Italy ranged between 2.2 and $6.4 \%$ vs. $0.35-0.5 \%$ in GP [27]. In a population-based relative risk estimation study, Màrild et al. found an overall 3-fold increased risk of CD in Swedish TS patients, ranging from 2 -fold in the first 5 years of life to a 5 -fold increase in females aged above 10 years at CD screening [28], which reinforces the fact that the prevalence of AD in TS evolves with age $[5,6]$.

The wide range in the prevalence of CD among TS series could be attributed to differences related to the age and sample-size of the population undergoing screening, the geographic location with ethnic variability in the frequency of predisposing human leukocyte antigen (HLA)-genotypes, variations in the serological testing, and the criteria used for CD diagnosis.

An outstanding feature of CD is that almost all CD patients carry genes coding for HLA-DQ2 and/or DR4$D Q 8$ heterodimers $[7,8]$. In the current study, the results of HLA-typing showed that all CD-seropositive girls presented the predisposing CD HLA-alleles as follows: case 1: $D Q A 1+/ D R B 1-$; case 2: DQA1+/DRB1+; and case 3: $D Q B 1+/ D R B 1-$. In agreement, the $2 \mathrm{CD}$-seropositive Brazilian girls with TS were positive for the predisposing HLA-DQ2 alleles and both had biopsy-confirmed CD [12].

The association between CD and IgA deficiency has been speculated to be due to either the shared HLA-types in both conditions or the failure to clear food antigens in IgA-deficient individuals that may trigger the onset of CD [29]. As regards this association, Bonamico et al. [10] dealt with a girl with TS who presented with IgA deficiency and CD. Mortensen et al. [4] reported that 3 out of 106 TS girls had IgA deficiency and showed 
variable seropositivity for IgG-based assays, and they all had negative endoscopic biopsies.

We detected 1 case with absolute IgA deficiency and 5 girls with partial IgA deficiency; they were all anti-tTG IgG-seronegative. In contrast, none of TS patients displayed abnormal levels of IgA during serological screening for CD in most of the previous studies [11, 12]. And in studies that examined immunological profile in TS patients, none of the Ig levels (IgA, IgG, and IgM) were lower than normal reference values for age [17].

Moreover, during screening for CD among general Egyptian children $(n=1500)$, Abu Zikrey et al. looked for IgA deficiency among 201 children who had anti-tTGIgA $\leq 5 \mathrm{U} / \mathrm{ml}$, but none of them was identified to have IgA deficiency [25].

As to the clinical features, CD in the current study appeared with typical gastrointestinal symptoms in case 1 and case 2 and was silent in case 3; to assess whether this girl will develop overt CD or will have rising anti-tTGIgA titre requires long-term follow-up.

A review of the literature revealed the fact that nearly a quarter of TS patients diagnosed with CD in most of screening studies were asymptomatic, and the remaining ones exhibited either typical or atypical symptoms of CD. This observation strengthens the rationale of periodic screening for CD in TS girls rather than investigating symptomatic patients, and the physician should have a high index of suspicion for CD in the presence of atypical features such as refractory iron deficiency anaemia.

Short stature is a universal phenotypic feature of TS [1], and it can also be a primary manifestation of monosymptomatic CD $[7,8]$. This observation reinforces the rationale for screening for both CD and TS in girls with short stature.

In this study, all TS girls with CD-seropositivity had lower height and weight compared to age-matched healthy Egyptian girls. However, in respect to Egyptian TS growth charts, only case 1 presented with stature below the $3^{\text {rd }}$ percentile, suggesting that screening for CD in TS girls should not only be performed in girls showing growth retardation in relation to TS curves.

Revision of medical files of TS girls with CD-seropositivity for the recorded growth velocities since the initiation of GHT revealed that case 1 presented with short stature and GH deficiency at the age of 6 years, at which point GHT was initiated at a dose of $0.09 \mathrm{IU} / \mathrm{kg} /$ day. Then the diagnosis of TS was established cytogenetically at the age of 6.5 years, which necessitated the increase of GHT dose to $0.15 \mathrm{IU} / \mathrm{kg} /$ day; growth velocity follow-up showed a significant growth deceleration after 7 years of age (from 6 to $2.5 \mathrm{~cm} /$ year). At the time of screening for CD case 1 was aged 8 years old, her height was $103 \mathrm{~cm}$ - which was below the $3^{\text {rd }}$ percentile with respect to Egyptian TS growth charts and was also more than 2 SD below the mid-parental target height $(161 \pm 5 \mathrm{~cm})$. In case 2 the growth velocity also decreased from 5.5 to $3.25 \mathrm{~cm} /$ year. The deceleration in growth velocities observed in case 1 and case 2 was concomitant with the appearance of gastrointestinal-related symptoms, indicating that undiagnosed gluten intolerance could be responsible, at least in part, for poor response to GHT, while case 3 did not exhibit any change in her growth velocity over the last year, which may have been related to $\mathrm{CD}$ and the fact that she was 14 years old, at which age the height potential could have been lost.

Further evaluation of TS girls with CD-seropositivity revealed that case 1 and case 2 had iron deficiency anaemia. All girls had low vitamin $\mathrm{D}$, elevated parathormone, and low or at risk of low BMD. The association between TS and CD could play a role in the observed comorbidities.

As to the frequency and concomitance of CD with previously diagnosed $\mathrm{AD}$, case 3 had coexisting seropositive-CD and AIT; however, the sequence of appearance of the 2 coexisting disorders cannot be predicted. In addition, 4 girls had isolated AIT. Similar to previous studies [2-5], AIT was the most common AD in our cohort, at $9 \%(5 / 55)$. They were all asymptomatic; however, they may develop overt thyroid dysfunction over time, emphasizing the importance of screening for thyroid dysfunction at TS diagnosis and thereafter annually regardless the symptoms [1].

Previous studies have reported higher frequency of T1DM among TS relative to GP, ${ }^{3}$ whereas others have not [2]. We detected only one TS girl (1.8\%) with T1DM, which, in itself, does not support the higher risk for developing T1DM in TS patients. We also detected one TS girl (1.8\%) with scalp psoriasis at the age of 14 years. Similarly, Wegiel et al. [13] reported psoriasis in 2 out of 134 TS patients $(1.5 \%)$ at the age of 5.9 and 16.9 years.

Some investigators reported an association between karyotype, particularly isochromosome and 45,X, and autoimmunity in TS patients $[4,6,26]$, whereas others were unable to establish such an association $[5,30]$. In the current TS cohort, the frequency of $\mathrm{AD}$ was higher in the 45 , $\mathrm{X}$ than in the non-45,X subgroup; nevertheless, it did not reach statistical significance, which may explained by the small sample size and older age of TS patients in previous studies $[5,6]$, based on the fact that autoimmunity in TS patients evolves over time $[5,6]$.

The impact of exposure to oestrogen on the overall prevalence of autoimmunity in the current TS cohort is not established. However, Wegiel et al. [13] detected a weak positive association between age at ERT and age at diagnosis of $\mathrm{AD}$.

Given that CD is frequently undiagnosed in clinical practice and failure of diagnosis of coexisting CD may predispose the affected individuals to increased risk of morbidity, mortality, and impaired quality of life, an active case finding approach is considered best practice $[7,8]$. The recent clinical practice guidelines for the care of girls and women with TS (2017) recommended 
that screening for CD in TS should start at the age of 2 years, and thereafter every 2 years [1]. Also, according to recent European Society Paediatric Gastroenterology, Hepatology, and Nutrition guidelines for diagnosing CD (2020), testing for total IgA and anti-tTG-IgA is recommended as initial screening in children with suspected $\mathrm{CD}$; the no-biopsy approach for CD diagnosis is considered in children with high serum anti-tTG-IgA values ( $\geq 10$ times the upper limit of normal) and endomysial antibodies positivity in a second serum sample; children with positive anti-tTG-IgA but lower titres $(<10$ times upper limit of normal) should undergo biopsies to reduce the risk of false positive diagnosis; and HLA- typing for $D Q 2 / D Q 8$ alleles is not required in children with high serum anti-tTG-IgA values and positive endomysial antibodies or in children with positive anti-tTG-IgA, if they qualify for CD diagnosis with biopsies [31].

The limitations of our study include its cross-sectional design and small sample size, which may overestimate the real prevalence rate of CD. However, our study is a single-centre study conducted on a paediatric TS cohort only with unified follow-up protocol. The diagnosis of CD was finally confirmed by the presence of specific HLA-heterodimer and/or duodenal biopsies.

\section{CONCLUSIONS}

Egyptian TS patients are a high-risk population for developing CD, with prevalence of $5.5 \%$, which is 12 -times higher than in the general population $(0.46 \%)$. All CD-seropositive girls displayed the predisposing CD HLA-alleles (DQ2 and/or DR4). CD could play a role in poor response to GHT, iron deficiency anaemia, and low BMD in CD-seropositive TS girls. Periodic screening for CD in TS girls allows for early recognition of CD and CD-comorbidities, and early initiation of a gluten-free diet along with management of associated nutritional deficiencies and adequate hormonal therapies at the appropriate age helps to achieve optimal health outcomes and best quality of life.

There is a desperate need for longitudinal studies to define the beneficial effects of a gluten-free diet on growth, nutritional status, $\mathrm{BMD}$, and long-term complications of CD in TS girls.

\section{ACKNOWLEDGMENTS}

We are grateful to all the families that participated in this study. We specially thank Staff members of Gastroenterology unit and Pathology unit at Mansoura University Children's Hospital for their cooperation.

\section{DISCLOSURE}

The authors declare no conflict of interest.

\section{REFERENCES}

1. Gravholt $\mathrm{CH}$, Andersen NH, Conway GS, et al.; International Turner Syndrome Consensus Group. Clinical practice guidelines for the care of girls and women with Turner syndrome: proceedings from the 2016 Cincinnati International Turner Syndrome Meeting. Eur J Endocrinol 2017; 177: G1-G70.

2. Bakalov VK, Gutin L, Cheng CM, et al. Autoimmune disorders in women with Turner syndrome and women with karyotypically normal primary ovarian insufficiency. J Autoimmun 2012; 38: 315-321.

3. Jorgensen KT, Rostgaard K, Bache I, et al. Autoimmune diseases in women with Turner's syndrome. Arthritis Rheum 2010; 62: 658-666.

4. Mortensen KH, Cleemann L, Hjerrild BE, et al. Increased prevalence of autoimmunity in Turner syndrome - influence of age. Clin Exp Immunol 2009; 156: 205-210.

5. El-Mansoury M, Bryman I, Berntorp K, et al. Hypothyroidism is common in Turner syndrome: results of a five-year follow-up. J Clin Endocrinol Metab 2005; 90: 2131-2135.

6. Elsheikh M, Wass JA, Conway GS. Autoimmune thyroid syndrome in women with Turner's syndrome- the association with karyotype. Clin Endocrinol (Oxf) 2001; 55: 223-226.

7. Hill ID, Dirks MH, Liptak GS, et al; North American Society for Pediatric Gastroenterology, Hepatology and Nutrition. Guideline for the diagnosis and treatment of celiac disease in children: recommendations of the North American Society for Pediatric Gastroenterology, Hepatology and Nutrition. J Pediatr Gastroenterol Nutr 2005; 40: 1-192.

8. Husby S, Koletzko S, Korponay-SzabÒ IR, et al.; ESPGHAN Working Group on Coeliac Disease Diagnosis; ESPGHAN Gastroenterology Committee; European Society for Pediatric Gastroenterology, Hepatology, and Nutrition. European Society for Pediatric Gastroenterology, Hepatology, and Nutrition guidelines for the diagnosis of coeliac disease. J Pediatr Gastroenterol Nutr 2012; 54: 136-160.

9. Thatcher N, Besser GM, Stephens AD. Turner's syndrome with coeliac disease, thin bones and abnormal liver function tests. Postgrad Med J 1973; 49: 738-740.

10. Bonamico M, Bottaro G, Pasquino AM, et al. Celiac disease and Turner syndrome. J Pediatr Gastroenterol Nutr 1998; 26: 496-499.

11. Moayeri H, Bahremand SH. Prevalence of celiac disease in patients with Turner's syndrome. Acta Med Iran 2005; 43: 287-290.

12. Dias Mdo C, Castro LC, Gandolfi L, et al. Screening for celiac disease among patients with Turner syndrome in Brasília, DF, midwest region of Brazil. Arq Gastroenterol 2010; 47: 246-249.

13. Wegiel M, Antosz A, Gieburowska J, et al. Autoimmunity Predisposition in Girls with Turner Syndrome. Front Endocrinol (Lausanne) 2019; 30: 511.

14. Nadeem M, Roche EF. Coeliac disease in Turner syndrome. Arch Dis Child 2013; 98: 649-650.

15. Rutigliano I, Sacco M, Pastore M, et al. Celiac disease and autoimmunity in Turner Syndrome. Digest Liver Dis 2015; 47: e268.

16. Su MA, Stenerson M, Liu W, et al. The role of X-linked FOXP3 in the autoimmune susceptibility of Turner Syndrome patients. Clin Immunol 2009; 131: 139-144.

17. Gawlik A, Berdej-Szczot E, Blat D, et al. Immunological profile and predisposition to autoimmunity in girls with Turner syndrome. Front Endocrinol 2018; 9: 307.

18. Saurer L, Mueller C. T cell mediated immunoregulation in the gastrointestinal tract. Allergy 2009; 64: 505-519.

19. El-Bassyouni HT, Afifi HH, Aglan MS, et al. Growth Curves of Egyptian Patients with Turner Syndrome. Am J Med Genet Part A 2012; 158A: 2687-2691. 
20. Aghamohammadi A, Cheraghi T, Gharagozlou M, et al. IgA deficiency: correlation between clinical and immunological phenotypes. J Clin Immunol 2009; 29: 130-136.

21. Kleigman RM, Behrman RE, Jenson HB, et al. Nelson Textbook of Pediatrics. 18th ed. Saunders Elsevier, Philadelphia 2007.

22. Oberhuber G, Granditsch G, Vogelsang H. The histopathology of coeliac disease: time for a standardised report scheme for pathologist. Eur J Gastroenterol Hepatol 1999; 11: 1185-1194.

23. Sacchetti L, Sarrantonio C, Pastore L, et al. Rapid identification of HLA DQA ${ }^{*} 0501, \mathrm{DQB1}^{*} 0201$ and DRB1 ${ }^{*} 04$ alleles in celiac disease by a PCR-based methodology. Clin Chem 1997; 43: 2204-2227.

24. Bianchi ML, Baim S, Bishop NJ, et al. Official positions of the International Society for Clinical Densitometry (ISCD) on DXA evaluation in children and adolescents. Pediatr Nephrol 2010; 25: 37-47.

25. Abu-Zekry M, Kryszak D, Diab M, et al. Prevalence of celiac disease in Egyptian children disputes the east-west agriculture dependent spread of the disease. J Pediatr Gastroenterol Nutr 2008; 47: 136-140.

26. Hamza RT, Raof NA, Abdallah KO. Prevalence of multiple forms of autoimmunity in Egyptian patients with Turner syndrome: relation to karyotype. J Pediatr Endocrinol Metab 2013; 26: 545-550.

27. Mazzanti L, Naeraa RW. Autoimmune diseases in Turner syndrome. Int Congr 2006; 1298: 42-48.

28. Mårild K, Størdal K, Hagman A, et al. Turner Syndrome and Celiac Disease: A Case Control Study. Pediatrics 2016; 137: e20152232

29. Cataldo E, Marino V, Bottaro G, et al. Celiac disease and selective immunoglobulin A deficiency. J Pediatr 1997; 131: 306-308.

30. Berglund A, Cleemann L, Oftedal BE, et al. 21-hydroxylase autoantibodies are more prevalent in Turner syndrome but without an association to the autoimmune polyendocrine syndrome type I. Clin Exp Immunol 2019; 195: 364-368.

31. Husby S, Koletzko S, Korponay-Szabó I, et al; European Society Paediatric Gastroenterology, Hepatology and Nutrition Guidelines for Diagnosing Coeliac Disease 2020. J Pediatr Gastroenterol Nutr 2020; 70: 141-156. 